\title{
Prospective Study of Drug-induced Interstitial Lung Disease in Advanced Breast Cancer Patients Receiving Everolimus Plus Exemestane
}

\author{
Annelieke E. C. A. B. Willemsen ${ }^{1}$ - Jolien Tol ${ }^{2}$ - Nielka P. van $\mathrm{Erp}^{3} \cdot$ Marianne A. Jonker $^{4} \cdot$ Maaike de Boer $^{5} \cdot$ Bob Meek $^{6}$. \\ Paul C. de Jong ${ }^{7}$. Coline van Moorsel ${ }^{6,8} \cdot$ Winald R. Gerritsen ${ }^{1} \cdot$ Jan C. Grutters ${ }^{6,8} \cdot$ Carla M. L. van Herpen ${ }^{1}$
}

Published online: 19 July 2019

(c) The Author(s) 2019

\begin{abstract}
Background Everolimus-related interstitial lung disease (ILD) (also: pneumonitis) poses a difficulty for physicians, as it is hard to discriminate ILD from other causes of respiratory symptoms and to decide on safe treatment continuation.

Objective We investigated the capability of pulmonary function tests (PFT), plasma biomarkers, everolimus pharmacokinetics, and FDG-PET to discriminate between everolimus-related ILD and other causes of respiratory problems and to predict the severity of ILD.

Patients and methods Women starting treatment with everolimus plus exemestane for advanced breast cancer were included. At baseline and during the first 3 months, respiratory symptoms, PFT with diffusion capacity of the lungs for carbon monoxide corrected for hemoglobin (DLCOc) and forced vital capacity, serum plasma biomarkers (including SP-D and YKL-40), everolimus trough concentration, and ${ }^{18}$ F-FDG-PET were prospectively recorded.

Results Twenty-seven (out of 29 included) patients were evaluable for analysis. Fifteen patients (56\%) developed everolimusrelated respiratory signs or symptoms and four patients $(15 \%)$ needed everolimus discontinuation and received corticosteroids. Change in DLCOc differentiated ILD from alternative diagnoses with 0.91 sensitivity and 0.78 specificity. Decrease in DLCOc (non-significant) was greatest in patients who needed everolimus discontinuation. Serum SP-D and YKL-40 could differentiate ILD from alternative diagnoses with 0.83 and 0.83 sensitivity, and 0.85 and 0.62 specificity, respectively. ${ }^{18}$ F-FDG-PET abnormalities did not precede clinical symptoms. No relationship between ILD and everolimus trough concentration was found.

Conclusions This study shows that everolimus-related ILD occurs frequently. Prospective monitoring of DLCOc in combination with measurement of serum SP-D and YKL-40 appear useful to discriminate ILD from other causes of respiratory symptoms. Clinicaltrials.gov identifier: NCT01978171.
\end{abstract}

\section{Introduction}

In the past decades many novel anti-cancer agents including targeted therapies and immune checkpoint inhibitors have been developed, resulting in significant improvement of treatment outcomes $[1,2]$. These new treatments are associated with specific toxicities, including pulmonary toxicities,

Electronic supplementary material The online version of this article (https://doi.org/10.1007/s11523-019-00656-2) contains supplementary material, which is available to authorized users.

Carla M. L. van Herpen

carla.vanherpen@ radboudumc.nl

Extended author information available on the last page of the article

\section{Key Points}

This is the first study to prospectively investigate druginduced interstitial lung disease and the value of diverse diagnostic biomarkers to discriminate ILD from alternate diagnoses.

Diffusion capacity and the plasma biomarkers YKL-40 and SP-D can differentiate everolimus-induced ILD from alternate causes.

A rapid decrease in diffusion capacity could be an indication of severe ILD needing treatment discontinuation. 
that require adequate management to prevent serious morbidity and even mortality [3,4]. Interstitial lung disease (ILD), or non-infectious pneumonitis, is an adverse event of special importance, as it is often hard to discriminate between different causes of respiratory symptoms as well as to decide on safe treatment continuation.

An anticancer drug that is associated with frequent occurrence of ILD is the mTOR inhibitor everolimus, with a reported incidence of ILD varying between 3 and 54\% [5]. In a meta-analysis of 4,242 cancer patients treated with mTOR inhibitors, the incidence of any grade pneumonitis was $11 \%$, any grade dyspnea $15 \%$, and any grade cough $23 \%$ [6]. It is important to note that mTOR inhibitor-induced ILD can be very serious, as respiratory insufficiency and even fatal cases have been described [7-9]. mTOR inhibitor-induced ILD is suspected to be of immunological origin, possibly by exposure of cryptic antigens, delayed-type hypersensitivity, or by an increase in proinflammatory cytokines [10-13].

Everolimus plus exemestane is a valuable treatment option for patients with advanced breast cancer (ABC), but ILD is amongst the most common high-grade adverse events and is a frequent cause of treatment discontinuation [14-17]. In the BOLERO- 2 trial, $10 \%$ of patients required everolimus dose interruption or reduction because of ILD [18].

Cough and dyspnea are reported frequently and may have several causes, including opportunistic infections such as Pneumocystis jirovecii pneumonia [19]. In patients with everolimus-induced ILD it is hard to predict whether or not treatment can be continued safely. Biomarkers leading to better recognition of ILD and to improved prediction of its severity are lacking, but would be of significant clinical value. Biomarkers that may contribute to this are pulmonary function tests (PFTs), plasma biomarkers, everolimus pharmacokinetics, and $\left[{ }^{18} \mathrm{~F}\right]$-fluorodeoxyglucose positron emission tomography with integrated computed tomography (FDG-PET).Two small retrospective studies have shown an association between mTOR inhibitor-induced ILD and a decline in diffusing capacity of the lung for carbon monoxide (DLCO) [20, 21]. In other ILDs, plasma biomarkers such as surfactant protein A (SP-A), surfactant protein D (SP-D), CC16, CCL18, YKL-40, lactate dehydrogenase (LDH), and cancer antigen (CA) 15-3 correlate with pulmonary inflammation [22-27]. Previous studies have demonstrated that a high everolimus trough concentration is associated with a higher risk of toxicity [14, 28]. Increased FDG-avidity in the lungs on FDG-PET scans has been reported in several patients with drug-induced ILD such as bleomycin, rituximab, etoposide, and paclitaxel, mostly as diffuse bilateral FDG-accumulation [29-35]. FDG-PET has been reported to detect ILD earlier than high resolution computed tomography [36]. Therefore, this may be an early marker of ILD.

It is not known yet if PFT can discriminate ILD from other causes or can predict its severity. Also, no biomarkers in the blood are known to predict or monitor the development and severity of drug-induced ILD. Furthermore, it is not known whether there is an exposure-effect relationship in the development of everolimus-induced ILD, as the pathophysiological mechanism of everolimus-induced ILD is not known.

A prospective analysis of any form of drug-induced ILD following patients from the start of treatment has not been performed yet. In the current study, we prospectively investigated in $\mathrm{ABC}$ patients receiving everolimus plus exemestane whether PFT, plasma biomarkers, everolimus trough concentrations, and FDG-PET could discriminate everolimusinduced ILD from other respiratory problems. Furthermore, we determined whether changes in PFT are indicative of the severity of ILD.

\section{Methods}

\subsection{Patients}

The present investigation (PREVENT study) was a prospective, open-label, observational, explorative study carried out in three hospitals in the Netherlands. The study protocol was approved by the Radboudumc ethics committee (reference number 2013-285) and all patients gave written informed consent. Clinicaltrials.gov identifier: NCT01978171.

We included postmenopausal women with $\mathrm{ABC}$ before the start of treatment with everolimus plus exemestane. Planned enrollment was 100 patients to guarantee the presence of enough cases of ILD. No formal power analysis was done as this was an exploratory study. Recruitment was considerably slower than anticipated due to reduced use of this treatment when data became available that no survival benefit was demonstrated. Furthermore, CDK4/6 inhibitors became available as an alternative line of treatment. For this reason and considering the high frequency of ILD already observed in the study participants, the study was closed early.

\subsection{Clinical Evaluation}

Patients were evaluated at baseline and at days $14,35,60$, 90, and 120 after start of therapy. At each visit adverse events were recorded using the Common Terminology Criteria for Adverse Events (CTCAE), version 4.03. Using clinical symptoms, PFT, and imaging, patients were classified as having airway disease, suspected ILD, ILD, or an alternative diagnosis (Table 1), as described in detail previously [5]. In case of respiratory symptoms, infectious analysis was performed with chest X-ray, sputum culture, 
PCR for respiratory viruses, serology for atypical pneumonia (Mycoplasma pneumoniae, Chlamydia psitacci), and legionella antigen test in urine. In case of possible opportunistic infection, a bronchoalveolar lavage for Pneumocystis jirovecii pneumonia was performed.

\subsection{Pulmonary Function Tests (PFTs)}

PFTs were performed according to ATS/ERS criteria at every scheduled visit until day 120 and additionally when respiratory symptoms occurred. Forced vital capacity (FVC in liters) and single breath diffusion capacity of the lungs for carbon monoxide (DLCO in $\mathrm{mmol} / \mathrm{min} / \mathrm{kPa}$ ) were measured. DLCO was corrected for hemoglobin (DLCOc) [37].

\subsection{Plasma Biomarkers}

Blood was drawn at every scheduled visit until day 120 and at the occurrence of new respiratory symptoms. More information on the measurements is provided in the Electronic Supplementary Methods. Considering the variation in absolute values between individuals, the ratio of plasma biomarkers relative to the baseline value was used.

\subsection{FDG-PET and Pharmacokinetics}

Patients could choose to participate in the FDG-PET imaging part or not. FDG-PET was performed at baseline, day
14, and day 35 after start of treatment. Scans were qualitatively judged by a nuclear medicine physician for FDG-avid abnormalities consistent with ILD. The temporal relationship between FDG-abnormalities and clinical symptoms was evaluated.

Everolimus trough concentrations were measured in whole blood at day 14, $24 \mathrm{~h}$ after everolimus administration, by a validated liquid chromatography tandem mass spectrometry technique.

\subsection{Statistics}

To compare groups of patients, for each patient we used the PFT or plasma biomarker measurement at the first moment of the patient's highest severity classification (ILD $>$ suspected ILD $>$ alternative diagnosis $>$ airway disease $>$ no respiratory signs). For patients without respiratory symptoms, the value of PFT closest to the moment of median onset of ILD was used (day 65). Data were analyzed qualitatively, as described in the Electronic Supplementary Methods.

Using a Mann-Whitney $U$ test, the change in PFT was compared between patients with everolimus-related (suspected) ILD and patients with respiratory signs due to an alternative diagnosis. Using a Mann-Whitney $U$ test, plasma biomarkers were compared between patients with (suspected) ILD and patients without respiratory symptoms, with airway disease, or with respiratory symptoms due to

Table 1 Diagnostic classification of respiratory symptoms [5]

\begin{tabular}{|c|c|c|c|c|}
\hline $\begin{array}{l}\text { CTCAE grade }{ }^{\mathrm{a}} \text { (dyspnea, } \\
\text { cough, fever) }\end{array}$ & PFT decline ${ }^{b}$ & $\begin{array}{l}\text { Chest CT parenchymal } \\
\text { abnormalities }\end{array}$ & Infectious analysis & Conclusion $^{\mathrm{c}}$ \\
\hline \multirow[t]{4}{*}{0} & \multirow[t]{2}{*}{ Non-significant } & Absent & - & No ILD \\
\hline & & Present & - & Suspected ILD \\
\hline & \multirow[t]{2}{*}{ Significant } & Absent & General & Inconclusive \\
\hline & & Present & General + consider BAL & ILD \\
\hline \multirow[t]{4}{*}{1 or 2} & \multirow[t]{2}{*}{ Non-significant } & Absent & General & Airway disease \\
\hline & & Present & General + consider BAL & Suspected ILD \\
\hline & \multirow[t]{2}{*}{ Significant } & Absent & General + consider BAL & Suspected ILD \\
\hline & & Present & General + BAL & ILD \\
\hline \multirow[t]{4}{*}{3 or 4} & \multirow[t]{2}{*}{ Non-significant } & Absent & General + consider BAL & Inconclusive \\
\hline & & Present & General + BAL & ILD \\
\hline & \multirow[t]{2}{*}{ Significant } & Absent & General + BAL & Inconclusive \\
\hline & & Present & General + BAL & ILD \\
\hline
\end{tabular}

CTCAE Common Terminology Criteria for Adverse Events, $P F T$ pulmonary function test, ILD interstitial lung disease, $B A L$ bronchoalveolar lavage, $D L C O c$ diffusion capacity of the lungs for carbon monoxide corrected for hemoglobin

${ }^{\mathrm{a} C T C A E}$ pneumonitis NOT included

${ }^{b}$ Significant PFT decline defined as: DLCOc corrected for Hb decline $\geq 2$ SD compared to baseline AND/OR FVC decline $\geq 2$ SD compared to baseline

${ }^{c}$ Provided that other possible diagnoses are considered or excluded, such as pulmonary infection, congestive heart failure, pulmonary embolism, carcinomatous lymphangitis or pleuritis, radiation recall pneumonitis, asthma bronchiale, or gastroesophageal reflux 
other causes. A receiver-operating-characteristic (ROC) curve analysis was performed, with calculation of the area under the curve (AUC), and testing whether this AUC significantly differed from 0.50 . An optimal cut-off value was determined using the Youden index (value with highest sum of sensitivity + specificity - 1) [38]. Sensitivity, specificity, and negative and positive predictive value were calculated.

In patients with (suspected) ILD, the change in DLCOc and $\mathrm{FVC}$ at the first moment of the highest classification was compared between patients with and without the need for everolimus discontinuation. In patients who discontinued everolimus due to (suspected) ILD, it was visually evaluated whether discontinuation was preceded by a rise in plasma biomarkers.

As this was an exploratory and hypothesis-generating analysis, no correction for multiple testing was performed.

\section{Results}

\subsection{Patients}

Between April 2014 and October 2017, 29 subjects were enrolled. Almost all patients who were eligible for the study did participate in the study. Two patients were a screen failure (in one patient after informed consent it was decided to start a different treatment and in the other patient informed consent was withdrawn due to a decline of general wellbeing), and 27 patients could be analyzed. Population demographics are described in Table 2.

\subsection{Respiratory Symptoms}

From the 27 patients included, 15 patients (56\%) developed everolimus-related respiratory problems (symptoms or DLCOc or CT abnormalities): three patients were classified as ILD, 11 with suspected ILD (two patients were asymptomatic, but with CT abnormalities consistent with ILD), and one with airway disease. Eight patients (30\%) had an alternative diagnosis (four progression of malignant pleural effusion, three suspected viral respiratory infection, one Pneumocystis jirovecii pneumonia). Four patients (15\%) had no respiratory symptoms. Details of the classification are described in Electronic Supplementary Table 1.

Median time to onset of (suspected) ILD was 65 days (range 32-119). Four patients discontinued treatment because of (suspected) ILD and were treated according to protocol with prednisolone $60 \mathrm{mg} /$ day followed by tapering, for a mean duration of 69 days (range 50-95) and everolimus was discontinued permanently. In three of these patients corticosteroids were started at the diagnosis of ILD and discontinuation of everolimus, and in one patient corticosteroids were started 10 days later. This resulted in clinical improvement in all cases. From the four patients who discontinued treatment because of (suspected) ILD, one patient had a history of pulmonary problems (asthma), one patient was a previous smoker, and one patient previously underwent radiation of the breast, but pulmonary abnormalities in this patient were bilateral. In these patients, there was a clear increase in pulmonary symptoms compared to baseline, therefore the symptoms were not attributable to an increase of the preexistent problems.

\subsection{PFTs}

Due to logistical reasons no PFTs were done in three patients at day 60 and in three patients at day 90. In the four patients who discontinued everolimus due to (suspected) ILD, DLCOc was better correlated with ILD than FVC. In three out of these four patients, $a \geq 2$ SD decrease in DLCOc preceded the diagnosis of ILD (Fig. 1). In two out of these four patients the PFT decline was considered significant according to the diagnostic protocol. No other patients had a significant PFT decline.

Table 2 Population demographics

\begin{tabular}{ll}
\hline Characteristic & \\
\hline Age (years; median and rage) & $62(35-76)$ \\
Race & All Caucasian \\
Eastern Cooperative Oncology Group Performance Status score \\
$\quad(n, \%)$ & $18 / 27(67)$ \\
0 & $8 / 27(30)$ \\
1 & $1 / 27(4)$ \\
2 & 95 \\
Follow-up time (days, median) & $8 / 27$ \\
Current or ex-smokers $(n)$ & 3 \\
History of pulmonary disease $(n)$ & 1 \\
Chronic obstructive pulmonary disease & 1 \\
Asthma bronchiale & 1 \\
Bronchiectases & $15 / 27(56 \%)$ \\
History of radiation of the breast region $(n, \%)$ & \\
Number of previous treatments (mean) & 1.1 \\
In adjuvant setting & 2.1 \\
In metastatic setting & 3.2 \\
Total & 1 patient at \\
Lymphocyte count $<500 / m m^{3}$ during treatment & two time- \\
& points \\
\hline
\end{tabular}




\subsubsection{PFTs to Discriminate (Suspected) Interstitial Lung Disease (ILD) from an Alternative Diagnosis in Patients with Respiratory Symptoms}

The median decrease in FVC was $0.21 \mathrm{~L}(8 \%)$ in the 14 patients with everolimus-related (suspected) ILD, compared to $0.20 \mathrm{~L}(6 \%)$ in the eight patients with respiratory symptoms due to an alternative cause ( $p=0.328$ and $p=0.328$, respectively). DLCOc decrease was $1.02 \mathrm{mmol} /$ $\mathrm{min} / \mathrm{kPa}(16 \%)$ in everolimus-related (suspected) ILD versus a $0.10 \mathrm{mmol} / \mathrm{min} / \mathrm{kPa}$ increase $(1 \%)$ in patients with other causes ( $p=0.004$ and $p=0.005$, respectively) (Fig. 2). ROC analysis using the absolute decrease in DLCOc to predict (suspected) ILD showed an AUC of $0.90(p=0.003)$. At the optimal cut-off point of a $0.16 \mathrm{mmol} / \mathrm{min} / \mathrm{kPa}$ decrease in DLCOc, this had a sensitivity of 0.92 and a specificity of 0.86 , with a positive and negative predictive value of 0.92 and 0.86 , respectively. Alternatively, if a cut-off of a $>0.0 \mathrm{mmol} / \mathrm{min} / \mathrm{kPa}$ DLCOc change is used to diagnose alternative causes and a $>1.0 \mathrm{mmol} / \mathrm{min} / \mathrm{kPa}$ DLCOc decrease for (suspected) ILD, 67\% of patients can be diagnosed and this is correct in $93 \%$ of cases.
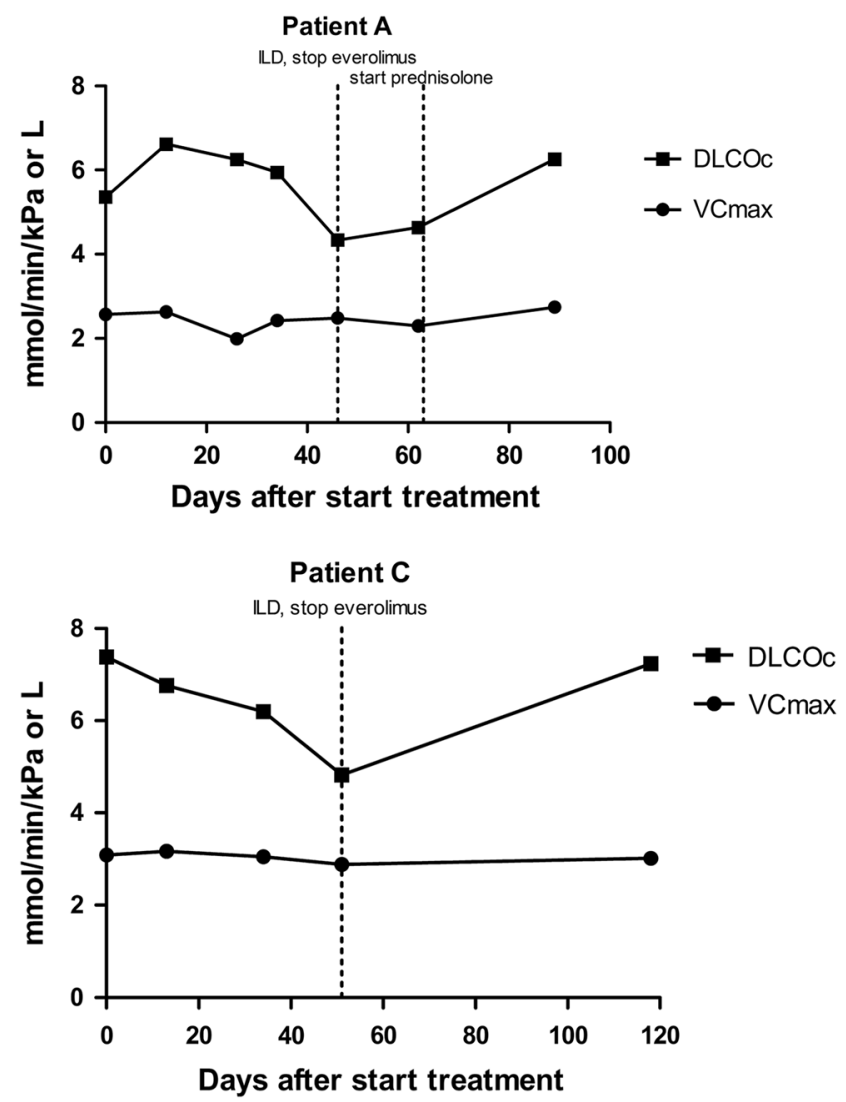

\subsubsection{PFTs to Predict the Need for Treatment Discontinuation in Patients with (Suspected) ILD}

Patients with (suspected) ILD with the need for treatment discontinuation showed a stronger DLCOc decrease compared to patients without the need for discontinuation (Fig. 3a, b), but this difference was not statistically significant. The median slopes of the 6 weeks before day zero were -0.042 and -0.00063 , respectively. However, the DLCOc decrease was not statistically significantly different in patients with $(-1.78 \mathrm{mmol} / \mathrm{min} / \mathrm{kPa}, 27 \%)$ versus without $(-0.73 \mathrm{mmol} / \mathrm{min} / \mathrm{kPa}, 14 \%)$ everolimus discontinuation ( $p=0.26$ and $p=0.15$, respectively). No significant difference was observed in FVC decrease in patients with $(0.21 \mathrm{~L}, 7 \%)$ or without everolimus discontinuation $(0.19 \mathrm{~L}, 8 \%),(p=0.70$ and $p=0.94$, respectively).

\subsubsection{Correlation between Diffusion Capacity of the Lungs For Carbon Monoxide Corrected for Hemoglobin (DLCOc) Decrease and Severity of Respiratory Symptoms}

In the total group of patients as well as in the group of patients with (suspected) ILD, no significant correlation
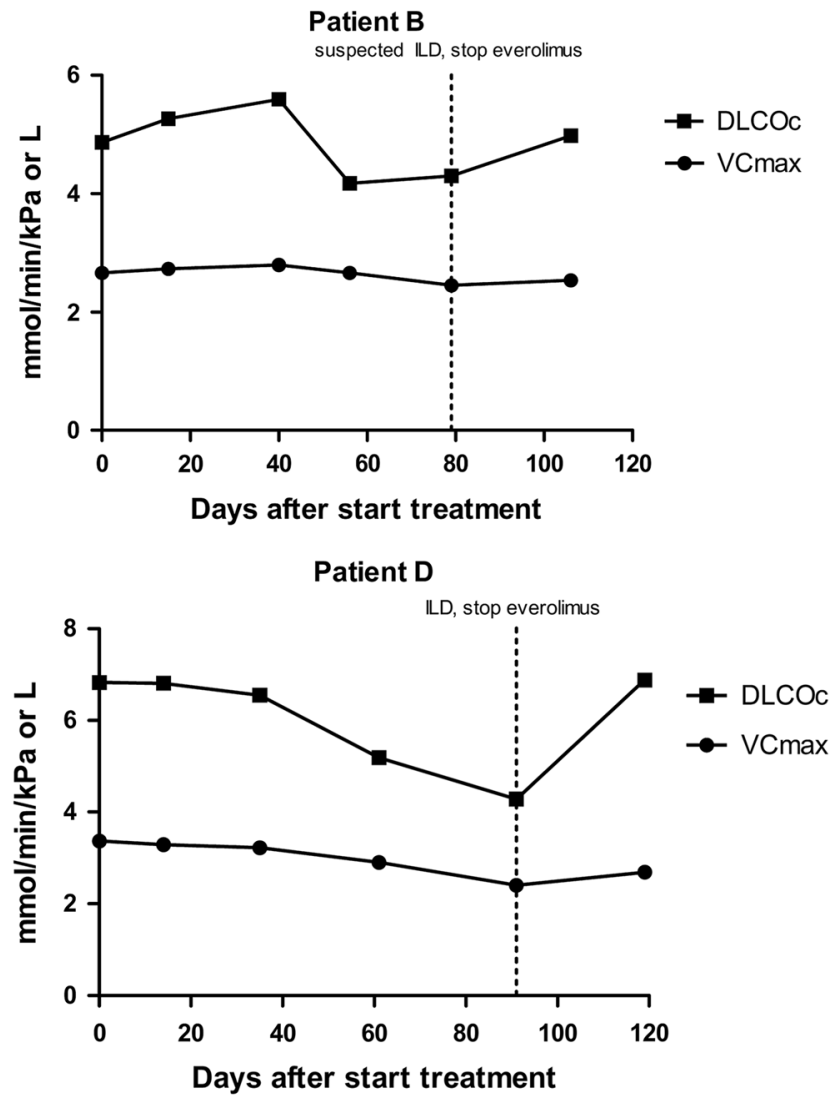

Fig. 1 Pulmonary function tests in patients who discontinued everolimus due to (suspected) interstitial lung disease (ILD) 
Fig. 2 Change in DLCOc at the first moment of the patient's highest severity classification compared to baseline per classification in patients with respiratory symptoms. Each bar represents one patient

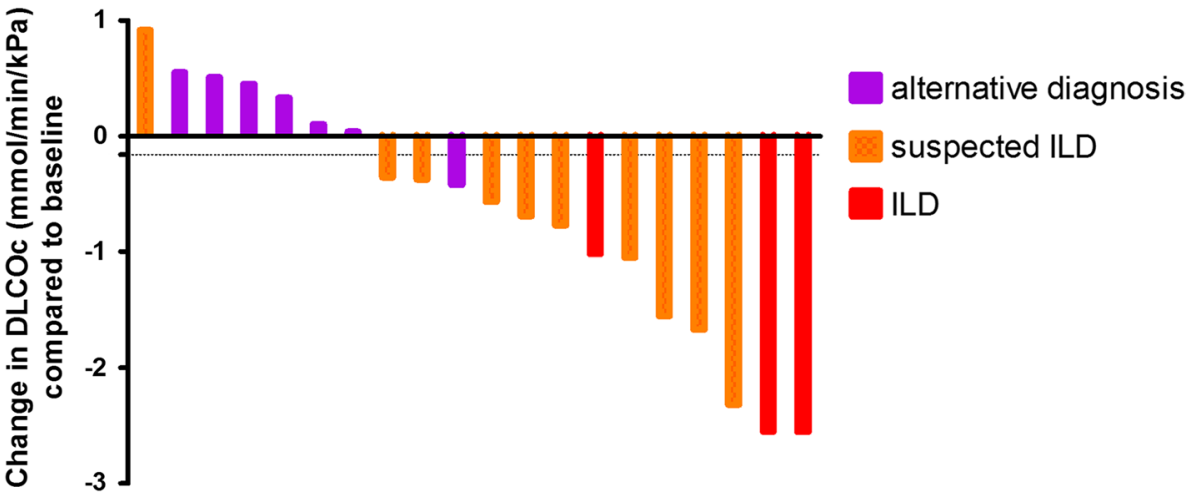

was observed between the severity of symptoms and DLCOc decrease, Spearman $r-0.26$ [95\% confidence interval (CI) $-0.60,0.16]$ and -0.44 (95\% CI - 0.80, 0.17).

\subsection{Plasma Biomarkers}

Plasma biomarkers were collected from 25 patients, with a total of 123 samples. Due to logistical problems, from two patients no samples could be collected and from the other 25 patients, one sample on day 14 and two samples on day 35 were not collected. One sample had to be discarded due to pre-analytical errors.

\subsubsection{Plasma Biomarkers to Discriminate Between Patients With and Without (Suspected) ILD}

Patients with (suspected) ILD were compared to patients without respiratory symptoms, airway disease, or respiratory symptoms due to an alternative cause. Concentrations of YKL-40 and SP-D in patients with (suspected) ILD were significantly higher compared to patients without (suspected) ILD (Table 2), median ratio 1.31 versus 0.94 and 1.27 versus 0.64 , respectively. No significant difference between the ILD and no-ILD group was seen regarding CA 15-3, uteroglobin, CCL18, SP-A, and LDH concentrations. YKL-40 and SP-D showed the highest ROC AUC. At the optimal cut-off points ( $>1.10$ and $>1.00$, respectively), these plasma biomarkers had high sensitivity and specificity and positive and negative predictive values (Table 3 ).

The optimal cut-offs for DLCOc and SP-D were combined, and patients were classified as with or without (suspected) ILD when both tests indicated the same direction. This could classify $70 \%$ of patients, with positive and negative predictive values of 1.0 and 0.80 , respectively.

\subsubsection{Plasma Biomarkers to Predict the Need for Treatment Discontinuation in Patients with (Suspected) ILD}

In three out of four patients who discontinued everolimus treatment due to ILD, plasma biomarker data were available. In these three patients, changes in concentration of plasma biomarkers SP-A, SP-D, and YKL-40 paralleled the development of ILD best (Fig. 4). However, plasma biomarker concentrations did not allow discrimination between patients with and without the need for treatment discontinuation. In patients with (suspected) ILD, the median ratio of YKL-40
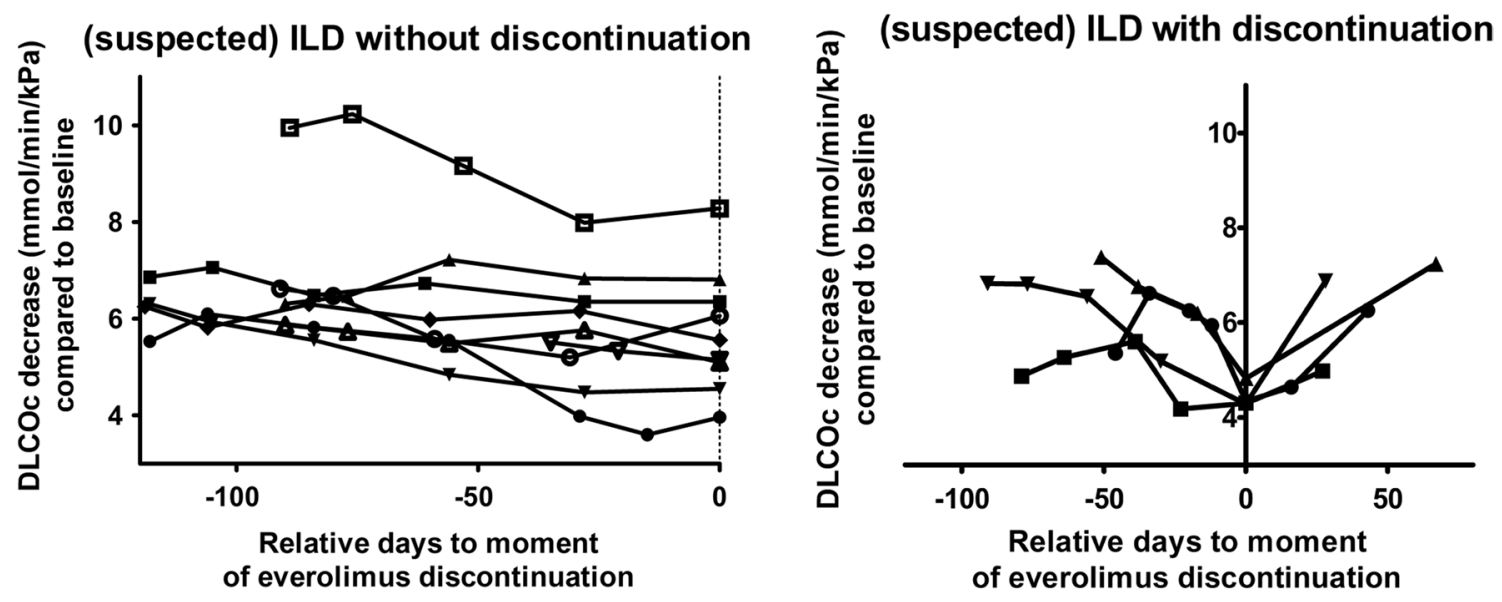

Fig. 3 Spaghetti plots of DLCOc for patients with (suspected) interstitial lung disease (ILD) with versus without the need for discontinuation 
was 1.29 in patients without the need for discontinuation, compared to 1.32 in the patients with the need for discontinuation $(p=1.00)$. For SP-D, medians were 1.09 and 1.52 , respectively $(p=0.11)$.

\subsection{Everolimus Pharmacokinetics}

Everolimus trough concentrations were measured in 26 patients. There was no difference in everolimus concentrations of patients with versus without (suspected) ILD, with geometric mean concentrations of 10.3 and $10.1 \mu \mathrm{g} / \mathrm{L}$, respectively $(p=0.94)$.

\subsection{FDG-PET}

Four patients did not participate in the FDG-PET imaging part, and therefore FDG-PET data were available from 11/15 patients with everolimus-related respiratory symptoms. From these patients, none had enhanced FDG uptake at day 14, while 7/11 patients showed an FDG-uptake pattern consistent with ILD on day 35. In these patients, FDG-uptake was most often focal consolidations, either unilateral or bilateral. From these seven patients with respiratory symptoms and FDG-PET abnormalities, six patients were classified as (suspected) ILD at or before day 35. The seventh patient showed pulmonary abnormalities consistent with ILD at day 35 and developed suspected ILD at day 89. As such, FDG-PET abnormalities mostly did not precede the clinical diagnosis. No enhanced FDG uptake was seen in patients without everolimus-related respiratory symptoms.

\section{Discussion}

To the best of our knowledge, this is the first prospective study investigating drug-induced ILD. We demonstrated that in $\mathrm{ABC}$ patients treated with everolimus, DLCOc decline and the plasma biomarkers YKL-40 and SP-D seem to discriminate patients with versus without (suspected) ILD. DLCOc shows a much better diagnostic performance for detecting ILD than FVC. The amount of DLCOc decline seems to be different for (suspected) ILD and alternative causes, such as malignant pleural effusion and respiratory infections. However, as DLCOc is foremost a marker of disease severity, analysis in more patients is needed to confirm the discriminative capability of DLCOc. A rapid decrease in absolute DLCOc could be an alarming indicator for severe toxicity necessitating treatment discontinuation. Furthermore, this study shows that the grade of respiratory symptoms is not a good reflection of the decrease in diffusion capacity. As such, measurement of DLCOc could be used as an indication of the severity of parenchymal changes. As the change in PFT compared to baseline is more informative than the absolute value, PFT is preferably performed at baseline and during follow-up.

SP-D is a protein involved in pulmonary immune and inflammatory regulation and in surfactant homeostasis, and has previously shown its value in hypersensitivity pneumonitis and idiopathic pulmonary fibrosis [39-41]. YKL40 is involved in tissue remodeling after an inflammatory response and has been associated with idiopathic pulmonary fibrosis, sarcoidosis, and hypersensitivity pneumonitis [42]. However, YKL-40 is also known to be associated with breast cancer markers [43]. We demonstrate that these proteins also increase in drug-induced ILD. SP-A, uteroglobin, CCL18, and LDH showed a non-significant increase in everolimusinduced ILD.

No correlation was seen between everolimus trough concentration and the development of (suspected) ILD, whereas other everolimus-related toxicities have been related to trough concentration $[14,28]$. This observation is in line with other studies that have indicated that the development of ILD is not exposure-related [44, 45]. Most patients with (suspected) ILD had FDG-avid pulmonary abnormalities.

Table 3 Diagnostic performance of change of plasma biomarkers from baseline to discriminate patients with and without (suspected) interstitial lung disease (ILD)

\begin{tabular}{|c|c|c|c|c|c|c|c|c|}
\hline Plasma biomarker & $\begin{array}{l}\text { Median ratio for } \\
\text { patients without (sus- } \\
\text { pected) ILD }(n=13)\end{array}$ & $\begin{array}{l}\text { Median ratio for } \\
\text { patients with (sus- } \\
\text { pected) ILD }(n=12)\end{array}$ & $p$ value & $\begin{array}{l}\text { Area under } \\
\text { the ROC } \\
\text { curve }\end{array}$ & Sensitivity & Specificity & $\begin{array}{l}\text { Positive } \\
\text { predictive } \\
\text { value }\end{array}$ & $\begin{array}{l}\text { Negative } \\
\text { predictive } \\
\text { value }\end{array}$ \\
\hline YKL-40 & 0.94 & 1.31 & 0.039 & 0.75 & 0.83 & 0.62 & 0.67 & 0.80 \\
\hline SP-D & 0.64 & 1.27 & 0.003 & 0.85 & 0.83 & 0.85 & 0.83 & 0.85 \\
\hline SP-A & 1.07 & 1.63 & 0.077 & 0.71 & & & & \\
\hline Uteroglobin & 1.23 & 1.29 & 0.849 & 0.53 & & & & \\
\hline CCL18 & 0.27 & 0.38 & 0.231 & 0.64 & & & & \\
\hline CA 15.3 & 1.19 & 1.02 & 0.479 & 0.59 & & & & \\
\hline LDH & 1.37 & 1.47 & 0.735 & 0.55 & & & & \\
\hline
\end{tabular}

$R O C$ receiver-operating characteristic 
Fig. 4 Plasma biomarkers in patients who discontinued everolimus due to (suspected) interstitial lung disease (ILD)
Patient A

(d46 ILD, stop everolimus)

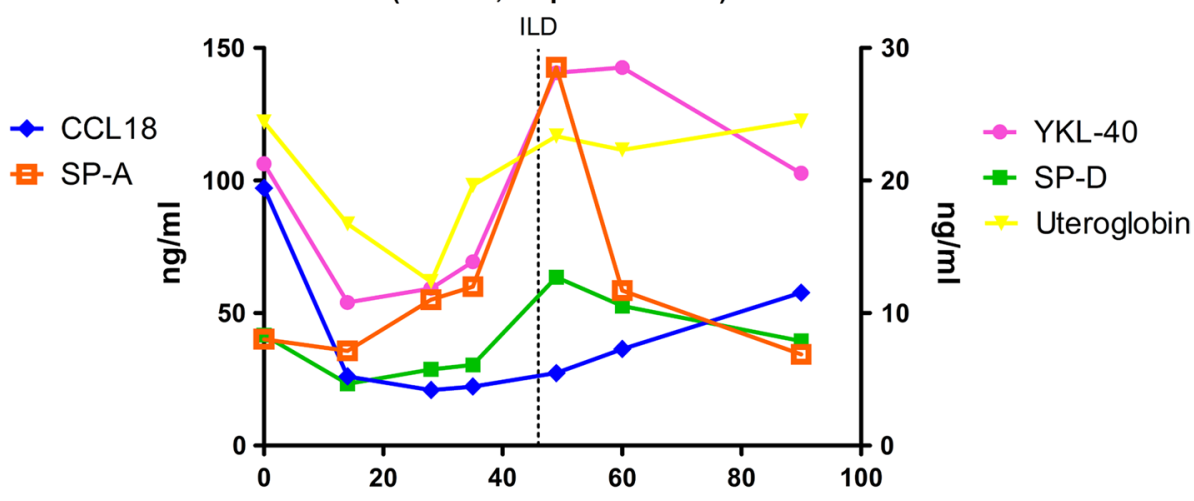

Patient B (d79 suspected ILD, stop everolimus)

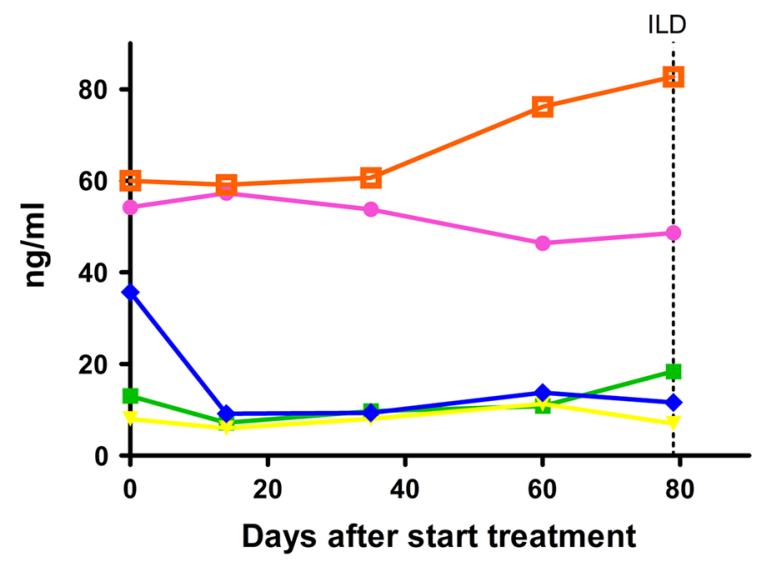

Patient C

(d51 ILD, stop everolimus, start prednisolone)

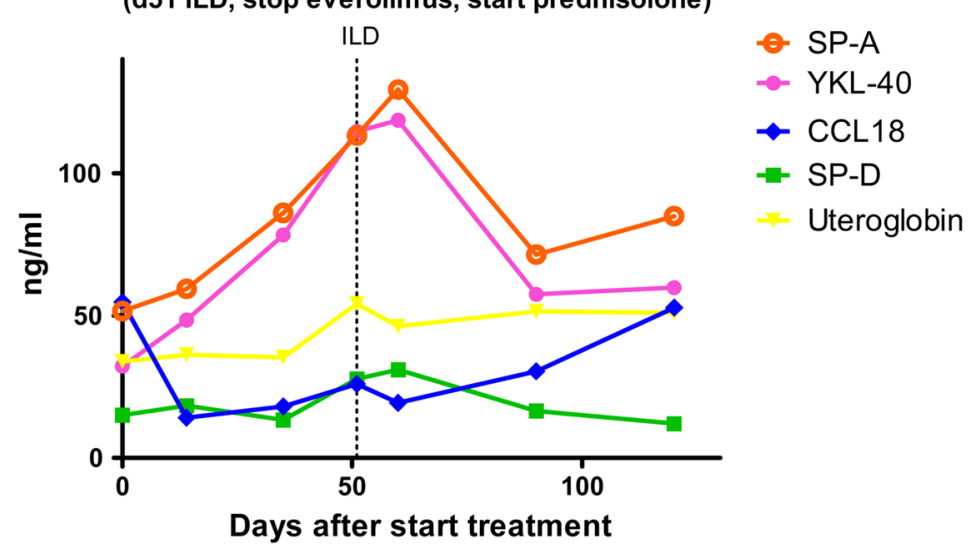

However, as these FDG-PET abnormalities mostly did not precede the clinical symptoms, FDG-PET does not seem to have significant predictive value for ILD.

The incidence of ILD was high, as $56 \%$ of patients developed everolimus-related respiratory problems, and $15 \%$ had to discontinue treatment because of drug-induced ILD. This high incidence is in contrast with earlier studies, such as the BOLERO-2 [16], which demonstrates that the incidence of ILD is higher when awareness is high. Furthermore, it illustrates that the incidence of ILD is very variable as there are no generally accepted criteria for its diagnosis. It is important to note that patients with CT abnormalities or a DLCOc decrease may have no or only minor symptoms and the majority of patients with suspected ILD continued everolimus without further deterioration. Everolimus discontinuation and treatment with prednisolone resulted in 
good recovery of symptoms and PFT in our patients with severe ILD.

The strong point of this study is the prospective collection of a comprehensive dataset. A prospective study of drug-induced ILD following patients from start of treatment has not been published before. A limitation of this study is the limited sample size. Despite this, however, clear observations could be made because of the high incidence of everolimus-induced ILD. Another limitation is that as yet the diagnosis of drug-induced ILD is largely made per exclusion. In patients with respiratory symptoms, analysis for viral, bacterial, and yeast infections was performed on clinical suspicion and therefore was not performed in all patients. As such, it is possible that cases have been assessed as ILD when an alternative diagnosis was missed. Also, the criteria we used have not yet been externally validated. However, to date, no validated criteria exist. The criteria we used are detailed and use multiple sources of information. Therefore, we believe this is preferable above for instance the CTCAE criteria, which are very non-specific. Furthermore, we acknowledge the risk that PFT is not a fully independent biomarker, as a significant PFT decline is part of the diagnostic criteria for ILD. However, only two patients had a significant PFT decline and patients were only diagnosed with ILD if other causes were excluded. In this study everolimus was used in combination with exemestane. Exemestane is not associated with ILD [46], and therefore this does not seem to influence ILD incidence.

Our data should be considered explorative and hypothesis-generating. Although our results will have to be confirmed in another study, we do suggest that a rise in SP-D combined with a decrease in DLCOc $(>0.16 \mathrm{mmol} / \mathrm{min} / \mathrm{kPa})$ is suggestive of everolimus-induced lung injury. DLCOc and plasma biomarker measurements could be useful in monitoring other forms of drug-induced ILD, especially for targeted therapies such as PI3 K inhibitors and Akt inhibitors, with similar class-related pneumonitis. However, the use of the biomarkers identified in our study need to be confirmed for these other anticancer therapies.

In summary, we report a high incidence of everolimusrelated ILD and have prospectively shown that DLCOc and SP-D and YKL-40 provide valuable tools for the early diagnosis of everolimus-induced lung toxicity. If after validation these tests can be applied in a clinical algorithm this could help to keep patients on treatment when possible, while also preventing drug-induced morbidity.

\section{Compliance with Ethical Standards}

Funding This study was partly funded by Novartis (2878/13).

Conflict of Interest Nielka P. van Erp and Carla M.L. van Herpen have received an Investigator Driven Research Grant from Novartis from which part of the study presented was paid. Annelieke E. C. A. B. Willemsen, Jolien Tol, Marianne A. Jonker, Maaike de Boer, Bob Meek, Paul C. de Jong, Coline van Moorsel, Winald R. Gerritsen, and Jan C. Grutters declare that they have no conflicts of interest that might be relevant to the contents of this article.

Open Access This article is distributed under the terms of the Creative Commons Attribution-NonCommercial 4.0 International License (http://creativecommons.org/licenses/by-nc/4.0/), which permits any noncommercial use, distribution, and reproduction in any medium, provided you give appropriate credit to the original author(s) and the source, provide a link to the Creative Commons license, and indicate if changes were made.

\section{References}

1. Shore ND. Advances in the understanding of cancer immunotherapy. BJU Int. 2015;116(3):321-9. https://doi.org/10.1111/ bju. 12692 .

2. Lee YT, Tan YJ, Oon CE. Molecular targeted therapy: treating cancer with specificity. Eur J Pharmacol. 2018;834:188-96. https ://doi.org/10.1016/j.ejphar.2018.07.034.

3. Teuwen LA, Van den Mooter T, Dirix L. Management of pulmonary toxicity associated with targeted anticancer therapies. Expert Opin Drug Metab Toxicol. 2015;11(11):1695-707. https ://doi.org/10.1517/17425255.2015.1080687.

4. Jain A, Shannon VR, Sheshadri A. Immune-related adverse events: pneumonitis. Adv Exp Med Biol. 2018;995:131-49. https ://doi.org/10.1007/978-3-030-02505-2_6.

5. Willemsen AE, Grutters JC, Gerritsen WR, van Erp NP, van Herpen CM, Tol J. mTOR inhibitor-induced interstitial lung disease in cancer patients: comprehensive review and a practical management algorithm. Int J Cancer. 2016;138(10):2312-21. https://doi. org/10.1002/ijc.29887.

6. Gartrell BA, Ying J, Sivendran S, Boucher KM, Choueiri TK, Sonpavde G, et al. Pulmonary complications with the use of mTOR inhibitors in targeted cancer therapy: a systematic review and meta-analysis. Target Oncol. 2014;9(3):195-204. https://doi. org/10.1007/s11523-013-0289-2.

7. White DA, Schwartz LH, Dimitrijevic S, Scala LD, Hayes W, Gross SH. Characterization of pneumonitis in patients with advanced non-small cell lung cancer treated with everolimus (RAD001). J Thorac Oncol. 2009;4(11):1357-63. https://doi. org/10.1097/JTO.0b013e3181ba20b1.

8. Yao JC, Shah MH, Ito T, Bohas CL, Wolin EM, Van Cutsem E, et al. Everolimus for advanced pancreatic neuroendocrine tumors. N Engl J Med. 2011;364(6):514-23. https://doi.org/10.1056/ NEJMoa1009290.

9. Saito Y, Gemma A. Current status of DILD in molecular targeted therapies. Int J Clin Oncol. 2012;17(6):534-41. https://doi. org/10.1007/s10147-012-0494-5.

10. Morelon E, Stern M, Israel-Biet D, Correas JM, Danel C, MamzerBruneel MF, et al. Characteristics of sirolimus-associated interstitial pneumonitis in renal transplant patients. Transplantation. 2001;72(5):787-90.

11. Pham PT, Pham PC, Danovitch GM, Ross DJ, Gritsch HA, Kendrick EA, et al. Sirolimus-associated pulmonary toxicity. Transplantation. 2004;77(8):1215-20.

12. Weichhart T, Costantino G, Poglitsch M, Rosner M, Zeyda M, Stuhlmeier KM, et al. The TSC-mTOR signaling pathway regulates the innate inflammatory response. Immunity. 2008;29(4):565-77. https://doi.org/10.1016/j.immun i.2008.08.012. 
13. Kezic A, Becker JU, Thaiss F. The effect of mTOR-inhibition on NF-kappaB activity in kidney ischemia-reperfusion injury in mice. Transplant Proc. 2013;45(5):1708-14. https://doi. org/10.1016/j.transproceed.2013.02.110.

14. Willemsen A, de Geus-Oei LF, de Boer M, Tol J, Kamm Y, de Jong PC, et al. Everolimus exposure and early metabolic response as predictors of treatment outcomes in breast cancer patients treated with everolimus and exemestane. Target Oncol. 2018;13(5):641-8. https://doi.org/10.1007/s11523-018-0596-8.

15. van den Eertwegh AJ, Karakiewicz P, Bavbek S, Rha SY, Bracarda $\mathrm{S}$, Bahl A, et al. Safety of everolimus by treatment duration in patients with advanced renal cell cancer in an expanded access program. Urology. 2013;81(1):143-9. https://doi.org/10.1016/j. urology.2012.09.019.

16. Baselga J, Campone M, Piccart M, Burris HA 3rd, Rugo HS, Sahmoud T, et al. Everolimus in postmenopausal hormonereceptor-positive advanced breast cancer. N Engl J Med. 2012;366(6):520-9. https://doi.org/10.1056/NEJMoa1109653.

17. Motzer RJ, Escudier B, Oudard S, Hutson TE, Porta C, Bracarda $\mathrm{S}$, et al. Phase 3 trial of everolimus for metastatic renal cell carcinoma: final results and analysis of prognostic factors. Cancer. 2010;116(18):4256-65. https://doi.org/10.1002/cncr.25219.

18. Ito Y, Noguchi S, Deleu I, Baselga J, Hortobagyi GN, Bachelot TD et al. Incidence, management, and resolution of noninfectious pneumonitis in BOLERO-2. J Clin Oncol. 2013;31(15):suppl; abstr 561

19. Willemsen AE, De Vos FY, Jansen A, de Boer M, Tjan-Heijnen VC, van Herpen CM. Diagnostic challenges of respiratory adverse events during everolimus treatment. Target Oncol. 2014;9(3):28791. https://doi.org/10.1007/s11523-014-0310-4.

20. Duran I, Siu LL, Oza AM, Chung TB, Sturgeon J, Townsley CA, et al. Characterisation of the lung toxicity of the cell cycle inhibitor temsirolimus. Eur J Cancer. 2006;42(12):1875-80. https://doi. org/10.1016/j.ejca.2006.03.015.

21. Park K, Kim HJ, Lee JL, Lee KH, Jeong IG, Song C, et al. Changes in the diffusion capacity for carbon monoxide and the development of non-infectious pneumonitis in patients with metastatic renal cell carcinoma treated with everolimus. Anticancer Res. 2014;34(10):5723-8.

22. Lee CG, Da Silva CA, Dela Cruz CS, Ahangari F, Ma B, Kang $\mathrm{MJ}$, et al. Role of chitin and chitinase/chitinase-like proteins in inflammation, tissue remodeling, and injury. Annu Rev Physiol. 2011;73:479-501. https://doi.org/10.1146/annurev-physiol-01211 $0-142250$.

23. Vij R, Noth I. Peripheral blood biomarkers in idiopathic pulmonary fibrosis. Transl Res. 2012;159(4):218-27. https://doi. org/10.1016/j.trsl.2012.01.012.

24. Hasegawa M, Fujimoto M, Hamaguchi Y, Matsushita T, Inoue K, Sato $\mathrm{S}$, et al. Use of serum clara cell $16-\mathrm{kDa}(\mathrm{CC} 16)$ levels as a potential indicator of active pulmonary fibrosis in systemic sclerosis. J Rheumatol. 2011;38(5):877-84. https://doi.org/10.3899/ jrheum.100591.

25. Kruit A, Gerritsen WB, Pot N, Grutters JC, van den Bosch JM, Ruven HJ. CA 15-3 as an alternative marker for KL-6 in fibrotic lung diseases. Sarcoidosis Vasc Diffuse Lung Dis. 2010;27(2):138-46.

26. Mizuno R, Asano K, Mikami S, Nagata H, Kaneko G, Oya M. Patterns of interstitial lung disease during everolimus treatment in patients with metastatic renal cell carcinoma. Jpn J Clin Oncol. 2012;42(5):442-6. https://doi.org/10.1093/jjco/hys033.

27. Kondo T, Hattori N, Ishikawa N, Murai H, Haruta Y, Hirohashi $\mathrm{N}$, et al. KL-6 concentration in pulmonary epithelial lining fluid is a useful prognostic indicator in patients with acute respiratory distress syndrome. Respir Res. 2011;12:32. https://doi. org/10.1186/1465-9921-12-32.
28. Deppenweiler M, Falkowski S, Saint-Marcoux F, Monchaud C, Picard N, Laroche ML, et al. Towards therapeutic drug monitoring of everolimus in cancer? Results of an exploratory study of exposure-effect relationship. Pharmacol Res. 2017;121:138-44. https://doi.org/10.1016/j.phrs.2017.04.029.

29. Buchler T, Bomanji J, Lee SM. FDG-PET in bleomycin-induced pneumonitis following ABVD chemotherapy for Hodgkin's disease-a useful tool for monitoring pulmonary toxicity and disease activity. Haematologica. 2007;92(11):e120-1. https://doi. org/10.3324/haematol.11856.

30. von Rohr L, Klaeser B, Joerger M, Kluckert T, Cerny T, Gillessen S. Increased pulmonary FDG uptake in bleomycinassociated pneumonitis. Onkologie. 2007;30(6):320-3. https:// doi.org/10.1159/000101517.

31. Falay O, Ozturk E, Bolukbasi Y, Gumus T, Ornek S, Ozbalak $\mathrm{M}$, et al. Use of fluorodeoxyglucose positron emission tomography for diagnosis of bleomycin-induced pneumonitis in Hodgkin lymphoma. Leuk Lymphoma. 2017;58(5):1114-22. https://doi. org/10.1080/10428194.2016.1236379.

32. Kalkanis D, Stefanovic A, Paes F, Escalon MP, Serafini A, Lossos IS. [18F]-fluorodeoxyglucose positron emission tomography combined with computed tomography detection of asymptomatic late pulmonary toxicity in patients with non-Hodgkin lymphoma treated with rituximab-containing chemotherapy. Leuk Lymphoma. 2009;50(6):904-11. https://doi.org/10.1080/1042819090 2919200.

33. Song BI, Lee SW, Lee HJ, Kang S, Jeong SY, Seo JH, et al. Rituximab-induced pneumonitis on F-18 FDG PET/CT in patient with non-Hodgkin lymphoma. Clin Nucl Med. 2010;35(8):601-3. https ://doi.org/10.1097/RLU.0b013e3181e4da5c.

34. Morikawa M, Demura Y, Mizuno S, Ameshima S, Ishizaki T, Okazawa $\mathrm{H}$. FDG positron emission tomography imaging of druginduced pneumonitis. Ann Nucl Med. 2008;22(4):335-8. https:// doi.org/10.1007/s12149-007-0109-9.

35. Post MC, Grutters JC, Verzijlbergen JF, Biesma DH. PET scintigraphy of etoposide-induced pulmonary toxicity. Clin Nucl Med. 2007;32(9):683-4. https://doi.org/10.1097/RLU.0b013e3181 23f78d.

36. Yamane T, Daimaru O, Ito S, Nagata T, Yoshiya K, Fukaya N, et al. Drug-induced pneumonitis detected earlier by 18F-FDGPET than by high-resolution CT: a case report with non-Hodgkin's lymphoma. Ann Nucl Med. 2008;22(8):719-22. https://doi. org/10.1007/s12149-008-0183-7.

37. American Thoracic Society. Single-breath carbon monoxide diffusing capacity (transfer factor). Recommendations for a standard technique-1995 update. Am J Respir Crit Care Med. 1995;152(6 Pt 1):2185-98. https://doi.org/10.1164/ajrccm.152.6.8520796.

38. Youden WJ. Index for rating diagnostic tests. Cancer. 1950;3(1):32-5.

39. Miyazaki Y, Tsutsui T, Inase N. Treatment and monitoring of hypersensitivity pneumonitis. Expert Rev Clin Immunol. 2016;12(9):953-62. https://doi.org/10.1080/17446 66X.2016.1182426.

40. Greene KE, King TE Jr, Kuroki Y, Bucher-Bartelson B, Hunninghake GW, Newman LS, et al. Serum surfactant proteins-A and -D as biomarkers in idiopathic pulmonary fibrosis. Eur Respir J. 2002;19(3):439-46.

41. Crouch EC. Surfactant protein-D and pulmonary host defense. Respir Res. 2000;1(2):93-108. https://doi.org/10.1186/rr19.

42. Long X, He X, Ohshimo S, Griese M, Sarria R, Guzman J, et al. Serum YKL-40 as predictor of outcome in hypersensitivity pneumonitis. Eur Respir J. 2017. https://doi.org/10.1183/13993 003.01924-2015.

43. Shao R, Cao QJ, Arenas RB, Bigelow C, Bentley B, Yan W. Breast cancer expression of YKL-40 correlates with tumour 
grade, poor differentiation, and other cancer markers. Br J Cancer. 2011;105(8):1203-9. https://doi.org/10.1038/bjc.2011.347.

44. Bouabdallah K, Ribrag V, Terriou L, Soria JC, Delarue R. Temsirolimus in the treatment of mantle cell lymphoma: frequency and management of adverse effects. Curr Opin Oncol. 2013;25(Suppl 2):S1-12. https://doi.org/10.1097/CCO.0b013e32835de8ee.

45. Atkins MB, Hidalgo M, Stadler WM, Logan TF, Dutcher JP, Hudes GR, et al. Randomized phase II study of multiple dose levels of CCI-779, a novel mammalian target of rapamycin kinase inhibitor, in patients with advanced refractory renal cell carcinoma. J Clin Oncol. 2004;22(5):909-18. https://doi.org/10.1200/ JCO.2004.08.185

46. van de Velde CJ, Rea D, Seynaeve C, Putter H, Hasenburg A, Vannetzel JM, et al. Adjuvant tamoxifen and exemestane in early breast cancer (TEAM): a randomised phase 3 trial. Lancet. 2011;377(9762):321-31. https://doi.org/10.1016/S0140 $-6736(10) 62312-4$.

\section{Affiliations}

\section{Annelieke E. C. A. B. Willemsen ${ }^{1}$. Jolien Tol ${ }^{2} \cdot$ Nielka P. van $\mathrm{Erp}^{3} \cdot$ Marianne A. Jonker $^{4} \cdot$ Maaike de Boer $^{5} \cdot$ Bob Meek $^{6}$. Paul C. de Jong ${ }^{7}$. Coline van Moorsel ${ }^{6,8}$. Winald R. Gerritsen ${ }^{1}$. Jan C. Grutters ${ }^{6,8}$. Carla M. L. van Herpen ${ }^{1}$}

1 Department of Medical Oncology, Radboud university medical center, route 452, P.O. Box 9101 , 6500 HB Nijmegen, The Netherlands

2

Department of Medical Oncology, Jeroen Bosch Hospital, P.O. Box 90153, 5200 ME ‘s Hertogenbosch, The Netherlands

3 Department of Pharmacy, Radboud university medical center, P.O. Box 9101, 6500 HB Nijmegen, The Netherlands

4 Department for Health Evidence, Radboud university medical center, P.O. Box 9101, 6500 HB Nijmegen, The Netherlands
5 Division of Medical Oncology Department of Internal Medicine, GROW-School of Oncology and Developmental Biology, Maastricht University Medical Center, Maastricht, The Netherlands

6 Department of Pulmonology, St. Antonius Hospital, Centre of Interstitial Lung Diseases, Nieuwegein, The Netherlands

7 Department of Medical Oncology, St. Antonius Hospital, Nieuwegein, The Netherlands

8 Division Heart \& Lungs, University Medical Center Utrecht, Utrecht, The Netherlands 